\title{
Interconnection between phase and amplitude parameters with anisotropy of Muller-matrix invariants
}

\author{
A.G. Ushenko, A.V. Dubolazov, V.A.Ushenko, Yu.A. Ushenko, M.Yu. Sakhnovskiy, \\ L.Y. Pidkamin, V.N. Balazyuk \\ Chernivtsi National University, 2 Kotsyubinsky Str., Chernivtsi, 58012,Ukraine; e-mail: a.dubolazov@chnu.edu.ua
}

\begin{abstract}
A new azimuthally stable polarimetric method for processing microscopic images of optically anisotropic structures of different biological layers prepared from histological sections has been proposed. A new model for definition of phase anisotropy inherent to biological tissues by using superposition of Mueller matrices of linear birefringence and optical activity has been proposed. The matrix element $M_{44}$ was chosen as the main information parameter, which value is independent of rotation angle of both sample and probing beam polarization plane.
\end{abstract}

Keywords: polarimetry, endometrium, laser image, filtering.

Manuscript received 05.07.16; revised version received 12.09.16; accepted for publication 16.11.16; published online 05.12.16.

\section{Theoretical basis of Muller-matrix imaging}

Development of computational methodologies for processing microscopic images is a new health-physical method. It is laser polarimetry of biological tissue histological sections [1-3]. It is based on measurement of the coordinate distributions (polarization maps) in the plane of polarization states of histological section microscopic images of biological tissues. This method allows a new, inaccessible to the histological and mathematical methods of analysis, information on the optical anisotropy (linear and circular birefringence) of multiscale structural elements inherent to various biological objects.

At the same time, a complex analysis of the polarization maps of a tissue specimen is azimuthally dependent on the probing beam polarization plane and sample rotation angle. This makes it difficult to use the method in comparative researching the groups of histological sections with different pathologies. Thus, further progress of laser polarimetry may be connected with the development of azimuthally stable methods of direct measuring the parameters of linear and circular birefringence.

Solution of this task can be reached using Muellermatrix cartography with the so-called rotational invariants. It has been shown in the works [4-6] that azimuthally stable, independent of the sample rotation angle $(\Theta)$ are the following matrix elements $M_{44} \Theta_{=}^{\prime}=$ const.

It has been determined that in the case of optically thin (attenuation coefficient $\tau \leq 0.1$ ) layers the $M_{44} \Theta$ value is preferably defined by phase anisotropy mechanisms - linear and circular birefringences [7-20].

$$
\begin{aligned}
& \text { Separation of these mechanisms } \\
& \left(M_{44} \Rightarrow\left(\begin{array}{c}
M_{44}-\text { linear } \\
M_{44}-\text { circular }
\end{array}\right)\right) \text { is possible by using spatial- } \\
& \text { frequency Fourier selection }[12,13] \text {. }
\end{aligned}
$$


Our research is aimed at designing the experimental method of azimuthally stable Fourier polarimetry and spatial-frequency selection of parameter distributions for linear and circular birefringence in the blood plasma donor films and patients with liver cirrhosis.

\subsection{The theory of method}

The following model concepts were offered by us [1-3] as the basis to analyze processes of modulation of laser radiation in the polycrystalline network of biological crystals with linear and circular birefringence:

- blood plasma consists of two optical anisotropic components - globulin and albumin crystals;

- the optical anisotropic albumin network is formed by large-scale (the range of the transverse dimensions $l \sim 30 \ldots 100 \mu \mathrm{m})$ crystals with a prevalence by linear birefringence;

- optical anisotropy of these structures is characterized by the distribution of the coordinate matrix element, where $\rho$ - direction of the optical axis, $\delta$ - phase shift between linearly polarized orthogonal components of the light beam amplitude;

- the optical anisotropic component of the globulin is formed by spherulitic crystals $(l \sim 5 \ldots 10 \mu \mathrm{m}, L \approx l)$ with predominantly circular birefringence $M_{44}$ where $\theta$ - polarization plane rotation angle.

\subsection{Optical realization of spatial-frequency Fourier} selection when studying endometrium

Fig. 1 presents a diagram of laser Fourier polarimeter with spatial-frequency filtration [9].

Illumination of a sample under study was performed by the parallel $\left(\varnothing=10^{4} \mu \mathrm{m}\right)$ beam of $\mathrm{He}-\mathrm{Ne}$ laser $1(\lambda=0.6328 \mu \mathrm{m}, W=5.0 \mathrm{~mW})$. The polarization light source consisted of quarter-wave plates $3 ; 5$ and polarizer 4 , it formed a right circularly polarized beam. Blood plasma films were placed in the focal plane of the polarization microobjective 7 (focal distance $-30 \mathrm{~mm}$, aperture -0.1 , magnification $-4 \times$ ). Behind the (Fourier) focal plane, the vignetting diaphragm was located, its size changed within the range of 2 to 300 pix. The polarization microobjective 8 (focal distance $-30 \mathrm{~mm}$, aperture -0.1 , magnification $-4 \times$ ) was located at the focal length from the frequency plane oflens 7 and, thus, performed inverse Fourier transform of a filtered out polarization field of laser radiation. The coordinate distribution of intensity of these fields, polarizationally filtered by quarter-wave plate 9 and polarizer 10 , was registered in the plane of CCD-camera 11 (The Imaging Source DMK 41AU02.AS, monochrome 1/2" CCD, Sony ICX205AL (progressive scan), resølution $1280 \times 960$, light sensitive area size $-7600 \times 6200 \mu \mathrm{m}$, sensitivity $-0.05 \mathrm{~lx}$, dynamic range -8 bit, SNR -9 bit, deviation of photosensitive characteristics from linear was no more than $15 \%$ ). It provided the range of measuring the structural elements in the polycrystalline network with the resolution of $2 . .2000 \mu \mathrm{m}$ [18-20].

The matrix element $M_{44}$ was calculated for each probing beam within every pixel $(m \times n)$

$$
M_{44}=\frac{I_{\otimes}^{\otimes}-I_{\oplus}^{\otimes}}{I_{\otimes}^{\otimes}+I_{\oplus}^{\otimes}}-0.5\left(\frac{I_{\otimes}^{0}-I_{\oplus}^{0}}{I_{\otimes}^{0}+I_{\oplus}^{0}}+\frac{I_{\otimes}^{90}-I_{\oplus}^{90}}{I_{\otimes}^{90}+I_{\oplus}^{90}}\right) .
$$

Here, $I_{\circledast}^{\otimes ; 0 ; 90}, \quad I_{\oplus}^{\otimes ; 0 ; 90}$ are the intensities of spatialfrequency filtered image $(\otimes ; \oplus)$ for each polarization state of probing beam $\left(\otimes ; 0^{\circ} ; 90^{\circ}\right)$.

To quantify the coordinate distributions, we used the statistical analysis [7-10]. We calculated a set of statistical moments of the 1-st to 4 -th orders $Z_{j=1 ; 2 ; 3 ; 4}$ by using the following algorithms

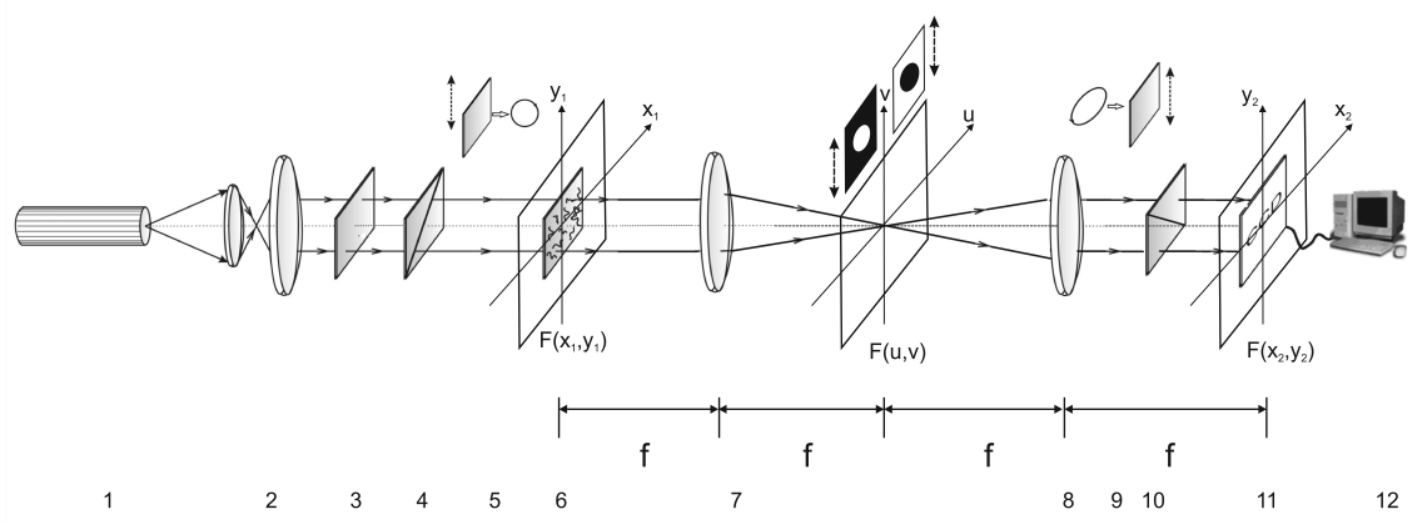

Fig. 1. Optical scheme of Fourier polarimeter: 1 - He-Ne laser; 2 - collimator; 3 - stationary quarter-wave plate; $5,9-$ mechanically movable quarter-wave plates; 4,10 - polarizer and analyzer, respectively; 6 - object of study; 7, 8 - polarization microobjectives; 11 - CCD camera; 12 - personal computer. $F\left(x_{1}, y_{1}\right)$ - object plane, $F\left(x_{2}, y_{2}\right)$-image plane, $F(u, v)-$ Fourier plane with the diaphragms. 


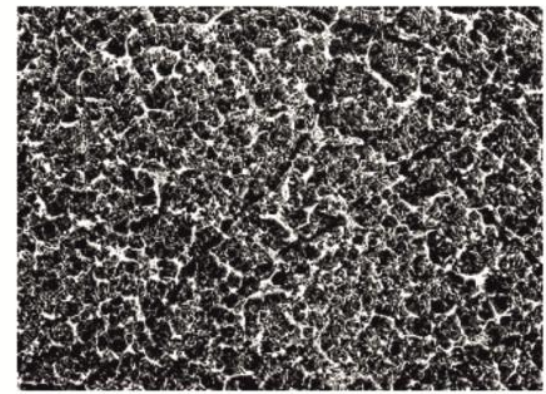

a)

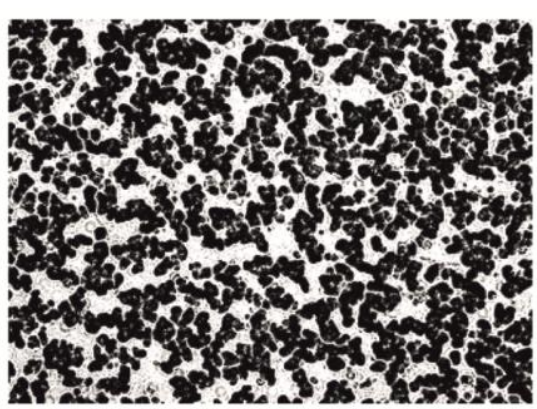

b)

Fig. 2. Microscopic images of the endometrium: group 1 (a) and group 2 (b).

$Z_{1}=\frac{1}{N} \sum_{i=1}^{N}\left|\mathbf{\varphi}_{i}^{\dagger}\right|, Z_{2}=\sqrt{\frac{1}{N} \sum_{i=1}^{N} \mathbf{q}_{i}^{2}}, Z_{3}=\frac{1}{\mathbf{\ell}_{2}{ }^{3}} \frac{1}{N} \sum_{i=1}^{N} \mathbf{q}_{\dot{i}}^{\Im}$,

$Z_{4}=\frac{1}{\boldsymbol{q}_{2}{ }^{4}} \frac{1}{N} \sum_{i=1}^{N} \mathbf{q}_{i}^{\mathbf{Z}}$.

The obtained data indicate the distribution histogram of random values optically anisotropic structures in the plane of histological section.

\section{Muller-matrix tomograms of birefringence observed in biological layers}

\subsection{Research objects}

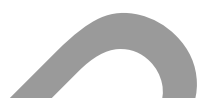

As objects for investigation, we chose two groups of optically-thin (attenuation coefficient $\tau \approx 0.087 \ldots 0.098$ ) single scattered endometrium donors (36 samples group 1) and patients with pathology (36 samples group 2).

The samples were prepared using a freezing microtome by using the standard technique. From the optical view point, these samples are characterized by transformation of polarization without depolarization. Fig. 2 shows the classic microscopic images for the samples of both groups. As can be seen, the coordinate large-scale structure of these classic microscopic images is similar. This fact makes it difficult to provide histological differentiation of endometrium.

\subsection{Spatial-frequency Fourier polarimetry of linear birefringence in endometrium}

For the purpose of choosing optimal conditions for spatial-frequency filtration, the following range $\Delta r=$ $2 . .50$ pix of possible sizes of the vignetting diaphragm was used.

The criterion for diaphragm size choosing is simultaneous change of the set of statistic moments

$Z_{j=1 ; 2 ; 3 ; 4}$ (4) of $M_{44}$. In our case, the optimal size was $\Delta r=30$ pix.
This geometric size was chosen for comparative investigations of optical anisotropy of the fibrilar networks in the endometrium tissue, which is characterized by coordinate distributions of rotational invariant $M_{44} \mathbf{S}_{-}$(Fig. 3).

A comparative analysis of aggregate parameters that characterize the matrix maps $M_{44} \boldsymbol{\phi}_{\text {, of linear }}$ birefringence for the large-scale optically anisotropic networks in the endometrium plasma samples revealed some discrepancies between them. Namely, the main extrema of histograms in the distributions of random

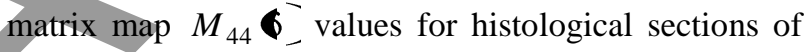
both types are localized in different areas. Thus, the most probable is value $M_{44} \sim 0.3$ for the group 1 (Fig. 3 (fragment 2)); for the sample $2 M_{44} \boldsymbol{\phi} \sim 0.6$ (Fig. 3 (fragment 4)). The revealed peculiarity, in our opinion, is related with a more developed structure of the endometrium typical for an ill patient. Quantitatively, the differences between matrix maps $M_{44} \boldsymbol{\phi}_{\text {, of the }}^{-}$ protein matrices corresponding to endometrium of both types are illustrated by the average values and standard deviations of the set of statistic $Z_{i=1 ; 2 ; 3 ; 4}$ parameters, which is summarized in Table 1. Also, let us introduce the specificity parameter $S p=a(a+b)^{-1}(a, b$ are the numbers of the correct and incorrect diagnoses within the group) in differentiation between healthy liver and that with cirrhosis.

Table 1. Parameters of the statistic structure of matrix maps $M_{44}(\delta)$ for linear birefringence in endometrium.

\begin{tabular}{|c|c|c|c|}
\hline \multirow{2}{*}{ Parameters } & \multicolumn{2}{|c|}{$M_{44}(\delta)$} & \multirow{2}{*}{$S p, \%$} \\
\cline { 2 - 3 } & normal & pathology & \\
\hline$Z_{1}$ & $0.25 \pm 0.059$ & $0.58 \pm 0.11$ & 80 \\
\hline$Z_{2}$ & $0.10 \pm 0.022$ & $0.14 \pm 0.031$ & 60 \\
\hline$Z_{3}$ & $1.23 \pm 0.37$ & $0.75 \pm 0.14$ & 85 \\
\hline$Z_{4}$ & $1.49 \pm 0.28$ & $1.06 \pm 0.19$ & 78 \\
\hline
\end{tabular}


A comparative analysis for the data of laserfrequency Fourier polarimetry of linear birefringence in endometrium of both groups of patients revealed sensitivity to differentiation of these states of all statistic moments of the $1^{\text {st }}$ to $4^{\text {th }}$ orders with the specificity level Se $\sim 78 \ldots 83 \%$ (printed in grey color in Table 2 ).

Table 2. Parameters of the statistic structure of matrix maps $M_{44}(\theta)$ for circular birefringence in endometrium.

\begin{tabular}{|c|c|c|c|}
\hline \multirow{2}{*}{ Parameters } & \multicolumn{2}{|c|}{$M_{44}(\theta)$} & \multirow{2}{*}{$S p, \%$} \\
\cline { 2 - 3 } & normal & pathology & \\
\hline$Z_{1}$ & $0.07 \pm 0.011$ & $0.10 \pm 0.017$ & 57 \\
\hline$Z_{2}$ & $0.12 \pm 0.018$ & $0.17 \pm 0.031$ & 62 \\
\hline$Z_{3}$ & $0.15 \pm 0.021$ & $0.49 \pm 0.39$ & 90 \\
\hline$Z_{4}$ & $0.34 \pm 0.043$ & $0.62 \pm 0.071$ & 80 \\
\hline
\end{tabular}

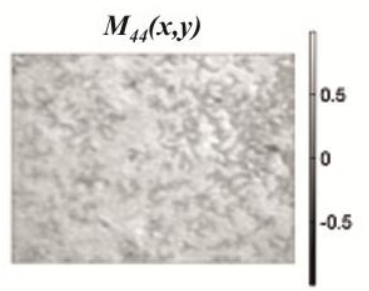

1)

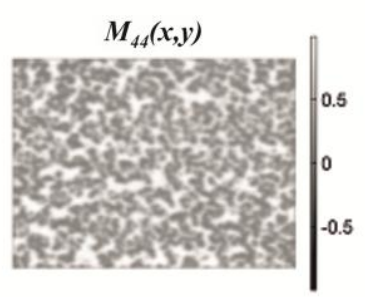

3)

\subsection{Spatial-frequency Fourier polarimetry of circular} birefringence in endometrium

Diagnostic possibilities to differentiate pathologically changed samples of endometrium tissue by using the method of high-frequency spatial-frequency Fourierdomain polarimetry of circular birefringence employing opaque filter illustrate probable dependences of matrix maps $M_{44}(\theta)$ adduced in the series in Fig. 4.

Comparing the findings about the structure of distributions for the circular birefringence index under conditions of high-frequency filtration of laser field radiation, it is noteworthy to emphasize range expansion of random values change $M_{44}(\theta)$ for the histogram of matrix map at the expense of a "pathologic" increase of the endometrium tissue.
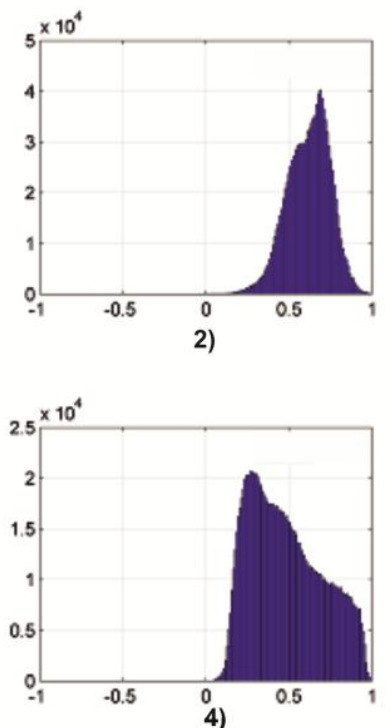

Fig. 3. "Low-frequency" coordinate maps $M_{44}(\delta)(1),(3)$ and histograms (2), (4) of the linear birefringence distribution of endometrium in the group $1((1),(2))$ and $2((3),(4))$.

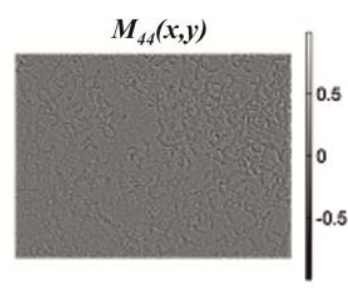

1)

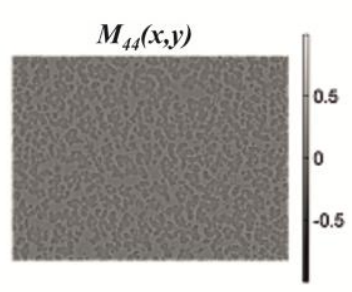

3)
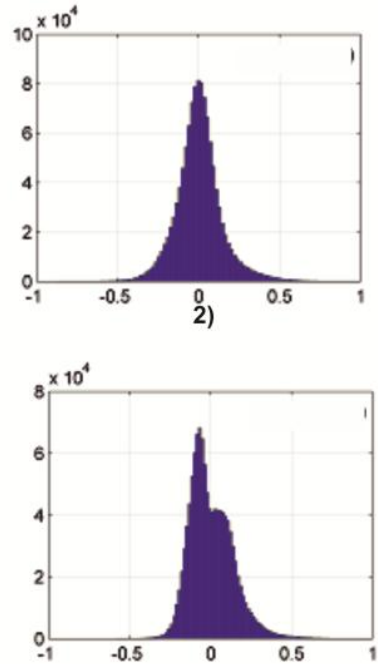

4)

Fig. 4. "High-frequency" coordinate maps $M_{44}(\theta)(1)$, (3) and histograms (2), (4) of the linear birefringence distribution typical for endometrium of the group $1((1),(2))$ and $2((3),(4))$. 
Quantitative differences between matrix maps $M_{44} \boldsymbol{\theta}_{-}^{-}$in the circular birefringence of polycrystalline collagenous networks of both types are illustrated by the average values and standard deviations of statistic set $Z_{i=1 ; 2 ; 3 ; 4}$ parameters, summarized in Table 2.

A comparative analysis revealed the following oncologic condition with a high level of specificity $(78 \leq \mathrm{Se} \leq 89 \%)$ of uterus parameters (depicted in grey color in Table 2):

- statistic moments of the $1^{\text {st }}$ and $2^{\text {nd }}$ of orders $\theta(n \times n$, the differences between their values is from 2 to 3.5 times.

\section{Conclusions}

A set of criteria for a polarization-phase differentiation of endometrium (normal and pathology) has been revealed and substantiated:

- $\quad$ statistical moments of the $1^{\text {st }}$ to $4^{\text {th }}$ orders that characterize distribution of azimuthally stable matrix element $M_{44} \boldsymbol{\phi}_{\text {, }}^{-}$related with linear birefringence in endometrium;

statistical moments of the $1^{\text {st }}$ to $4^{\text {th }}$ orders that characterize distribution of azimuthally stable matrix element $M_{44} \theta_{\text {, }}^{-}$related with circular birefringence in endometrium.

\section{Acknowledgement}

This work was supported by the grants №0116U001446, №0116U001449, №0115U003241, №0115U003227, №0115U003235 from the Ukraine Foundation for Basic Research.

\section{References}

1. O.V. Angelsky, C.Y. Zenkova, M.P. Gorsky, N.V. Gorodynśka, Feasibility of estimating the degree of coherence of waves at the near field // Appl. Opt. 48(15), p. 2784-2788 (2009).

2. O.V. Angel'skii, A.G. Ushenko, A.D. Arkhelyuk, S.B. Ermolenko, D.N. Burkovets, Structure of matrices for the transformation of laser radiation by biofractals // Quantum Electronics, 29(12), p. 1074-1077 (1999).

O.V. Angelsky, R.N. Besaha, A.I. Mokhun, I.I. Mokhun, M.O. Sopin, M.S. Soskin, M.V. Vasnetsov // Singularities in vectoral fields // Proc. SPIE, 3904, p. 40 (1999).

4. Yu.A. Ushenko, T.M. Boychuk, V.T. Bachynsky, O.P. Mincer, Diagnostics of structure and physiological state of birefringent biological tissues: Statistical, correlation and topological approaches, in: Handbook of Coherent-Domain Optical Methods. Springer Science+Business Media, New York, 2013, p. 107-148.
5. J.M. Bueno, J. Jaronski, Spatially resolved polarization properties for in vitro corneas // Ophthal., Physiol. Opt. 21, No. 5, p. 384-392 (2001).

6. M. Shribak and R. Oldenbourg, Techniques for fast and sensitive measurements of two-dimensional birefringence distributions // Appl. Opt. $\mathbf{4 2}$ p. 3009-3017 (2003).

7. M.H. Smith, Interpreting Mueller matrix images of tissues // Proc. SPIE, 4257, p. 82-89 (2001).

8. V.A. Holovatsky, O.M. Makhanets, O.M. Voitsekhivska, Oscillator strengths of electron quantum transitions in spherical nanosystems with donor impurity in the center /I Physica E: Low-Dimension. Systems and Nanostruct. 41(8), p. 1522-1526(2009).

9. R. Jóźwicki, K. Patorskí, O.V. Angelsky, A.G. Ushenko, D.N. Burkovets, Automatic polarimetric system for early medical diagnosis by biotissue testing // Optica Applicata, 32(4), p. 603612 (2005).

10. J.W. Goodman, Statistical properties of laser speckle patters, in: Laser Speckle and Related Phenomena. Ed. J.C. Dainty. Berlin, SpringerVerlag, 1975, p. 9-75.

11. Yu.A. Ushenko, Yu.Ya. Tomka, A.V. Dubolazov, Laser diagnostics of anisotropy in birefringent networks of biological tissues in different physiological conditions // Quantum Electronics, 41(2), p. 170-175 (2011).

12. V.A. Ushenko, M.I. Sidor, Y.F. Marchuk, N.V. Pashkovskaya, D.R. Andreichuk, Azimuthinvariant Mueller-matrix differentiation of the optical anisotropy of biological tissues // Optics and Spectroscopy, 117(1), p. 152-157 (2014).

13. V.A. Ushenko, N.I. Zabolotna, S.V. Pavlov, D.M. Burcovets, O.Yu. Novakovska, Muellermatrices polarization selection of two-dimensional linear and circular birefringence images // Proc. SPIE, 9066, Eleventh International Conference on Correlation Optics, 90661X (2013).

14. V.A. Ushenko, M.P. Gorsky, Complex degree of mutual anisotropy of linear birefringence and optical activity of biological tissues in diagnostics of prostate cancer // Optics and Spectroscopy, 115(2), p. 290-297 (2013).

15. Y.A. Ushenko, M.P. Gorsky, A.V. Dubolazov, A.V. Motrich, V.A. Ushenko, M.I. Sidor, A.O. Karachevtsev, Spatial-frequency Fourier polarimetry of the complex degree of mutual anisotropy of linear and circular birefringence in the diagnostics of oncological changes in morphological structure of biological tissues // Quantum Electronics, 42(8), p. 727 (2012).

16. V.A. Ushenko, Complex degree of mutual coherence of biological liquids, in: ROMOPTO International Conference on Micro-to NanoPhotonics III (pp. 88820V-88820V). International Society for Optics and Photonics (2013). 
17. Y.O. Ushenko, O.V. Dubolazov, A.O. Karachevtsev, M.P. Gorsky, Y.F. Marchuk, Wavelet analysis of Fourier polarized images of the human bile // Appl. Opt. 51(10), p. C133-C139 (2012).

18. Y.A. Ushenko, V.A. Ushenko, A.V. Dubolazov, V.O. Balanetskaya, N.I. Zabolotna, Mueller-matrix diagnostics of optical properties of polycrystalline networks of human blood plasma // Optics and Spectroscopy, 112(6), p. 884-892 (2012).
19. Yu.A. Ushenko, Yu.Ya. Tomka, A.V. Dubolazov, Laser diagnostics of anisotropy in birefringent networks of biological tissues in different physiological conditions // Quantum Electronics, 41(2), p. 170-175 (2011).

20. Y.A. Ushenko, A.V. Dubolazov, V.O. Balanetskaya, A.O. Karachevtsev, V.A. Ushenko, Waveletanalysis of polarization maps of human blood plasma // Optics and Spectroscopy, 113(3), p. 332343 (2012).

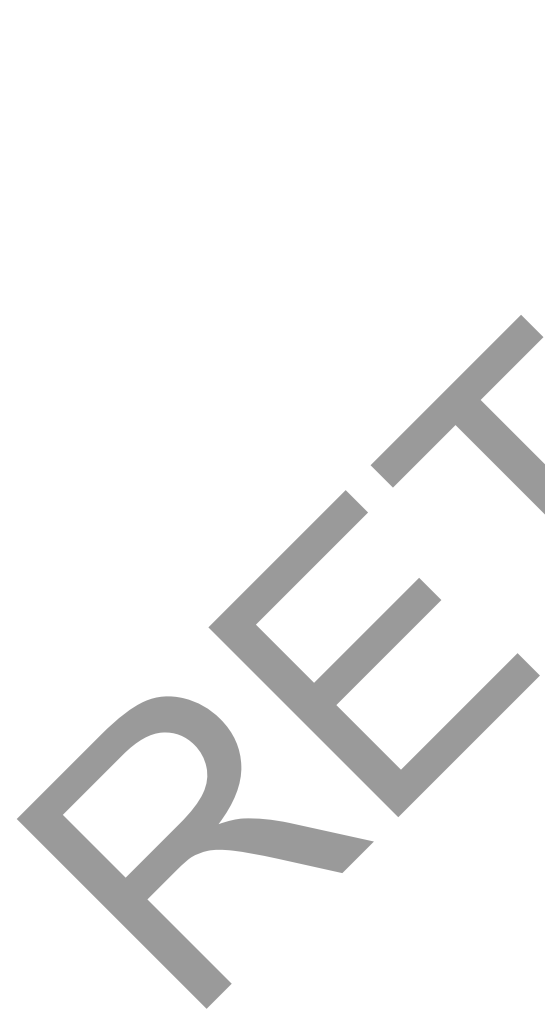

